\title{
A Soft Landing Trajectory Design and Control Strategy
}

\author{
Haiyan Xuan ${ }^{1, a}$, Yan Sun ${ }^{2, b}$, Peijian Yan ${ }^{3, c}$, Shaofei Dong, ${ }^{4, d}$, Panhong Zhao ${ }^{3,}$ \\ ${ }^{1}$ School of Economics and Management, Lanzhou University of Technology, Lanzhou 730050, \\ China; \\ ${ }^{2}$ School of Science, Lanzhou University of Technology, Lanzhou 730050, China; \\ ${ }^{3}$ College of Petrochemical Engineering, Lanzhou University of Technology, Lanzhou 730050, \\ China; \\ ${ }^{4}$ School of Materials Science and Engineering, Lanzhou University of Technology, Lanzhou 730050, \\ China; \\ ahaiyanxuan@gmail.com, byansun6@163.com, \\ cyanpj183@163.com, dongsf888@163.com, ${ }^{\mathrm{e}}$ zhaoph163@163.com
}

Keywords: Chang' e 3, soft landing, trajectory design, optimum control.

\begin{abstract}
This paper aims at realizing a soft landing of Chang' e 3 in a predetermined region on the moon. In line with the principle of low cost and business-friendly. It according to the scheduled landing point and combines with the actual operation of the Chang' e 3's track to put forward the scheme of 'safe radius spiral search algorithm' and finally gives the landing trajectory and optimal control strategy.
\end{abstract}

\section{Introduction}

Chang' e 3 lunar exploration satellite was successfully launched marks China's scientific and technological level has reached a peak. Many scholars at home and abroad were committed to the research of a soft landing orbit design and control strategy, such as Xu Min[1] proposed Pontryagin maximum principle to analyze minimum fuel orbit control problems and obtained the optimal braking maneuver control law; Pieson B L[2] studied the optimal control problems of the first three track section. Literature [3] and [4] make use of frozen orbit to design the lunar satellite orbit.

The lunar soft landing requires orbiter's relative velocity is very small (less than $6 \mathrm{~m} / \mathrm{s}$ )[5] when landing on the moon surface and the key is landing point selection. So in this paper, we identify a landing point firstly and calculate the position in the perilune and apolune with the movement mechanism of Chang 'e 3 landing at the landing orbit and relevant data. Taking into account the elevation is very low when Chang 'e 3 in the perilune, so we will consider its landing on the orbital motion as a motion model on the horizontal plane on the moon. Meanwhile, we decompose it's motion into two directions with vertical and horizontal and on the basis of the principle of minimizing fuel consumption and economic and efficient[6] to design a soft landing optimal control strategy finally.

\section{Position in the perilune and the apolune}

To determine the position in the perilune and the apolune, we should know about landing prepared orbit and corresponding data firstly.

From the information[5] we know: The operation quality of Chang 'e 3 in the landing prepared orbit is $2.4 t$, the main reduction of engine which installed in the under part is able to produce the adjustable thrust from $1500 \mathrm{~N}$ to $7500 \mathrm{~N}$, and specific impulse is $2940 \mathrm{~m} / \mathrm{s}$ which can content the control requirement in adjusting speed. Chang' e 3 will descend in the form of parabola in the perilune which is 15 kilometers that is called " black 750 seconds ". Scheduled landing site of Chang'e 3 is $19.51 \mathrm{~W}, 44.12 \mathrm{~N}$ and the altitude is $-2641 \mathrm{~m}$. Landing prepared orbit is an elliptical 
orbit with the distance of $15 \mathrm{~km}$ in the perilune and $100 \mathrm{~km}$ in the apolune. Landing trajectory has six stages between the perilune to the landing site and a soft landing process, the first three stages are deceleration section, rapid adjustment and coarse obstacle avoidance respectively[7].

In order to do the research successfully, we do five hypotheses: 1: the moon is a ball; 2: Chang 'e 3 in the same longitude planar motion in the first three stages before landing and look the moon surface as plane in the second and third stage; 3: in the first three phases, Chang 'e 3 has a constant reverse thrust $F_{t}$ in horizontal direction; 4: ignore the moon's auto biography and the gravity.

According to the hypothesis 2, we know the perilune, the apolune and scheduled landing site in the same longitude line. In the Fig. $1, O$ represents selenocentric coordinate, axis $x$ points to the North Pole, axis $y$ is in the equatorial plane. The trajectory of Chang 'e 3 is curve $A B C$ in Fig. 2 in the second and third phase.

Assume $F_{t}$ : constant reverse thrust; $\dot{m}$ : fuel consumption kilograms per unit time; $m(t)$ : quality of Chang 'e 3 in the landing orbit at moment $t$, initial mass $m_{0}=2400 \mathrm{~kg} ; R$ : radius of the moon $(1737.013 \mathrm{~km})$; ${ }^{s(t)}$ : horizontal movement of Chang 'e 3 in the landing orbit at moment $t$; $v_{e}$ : specific impulse $(2940 \mathrm{~m} / \mathrm{s}) ; t: t=0$ is initial point in the second phase; $v(t)$ : horizontal velocity of Chang 'e 3 in the landing orbit at moment $t$, initial velocity $v_{0}=1700 \mathrm{~m} / \mathrm{s} ; \mathrm{a}(t)$ : acceleration of Chang 'e 3 at $t$ moment; $\alpha$ : scheduled landing site latitude $(44.12 N)$; $\beta_{0}$ : difference of latitude between scheduled landing site and the perilune latitude.
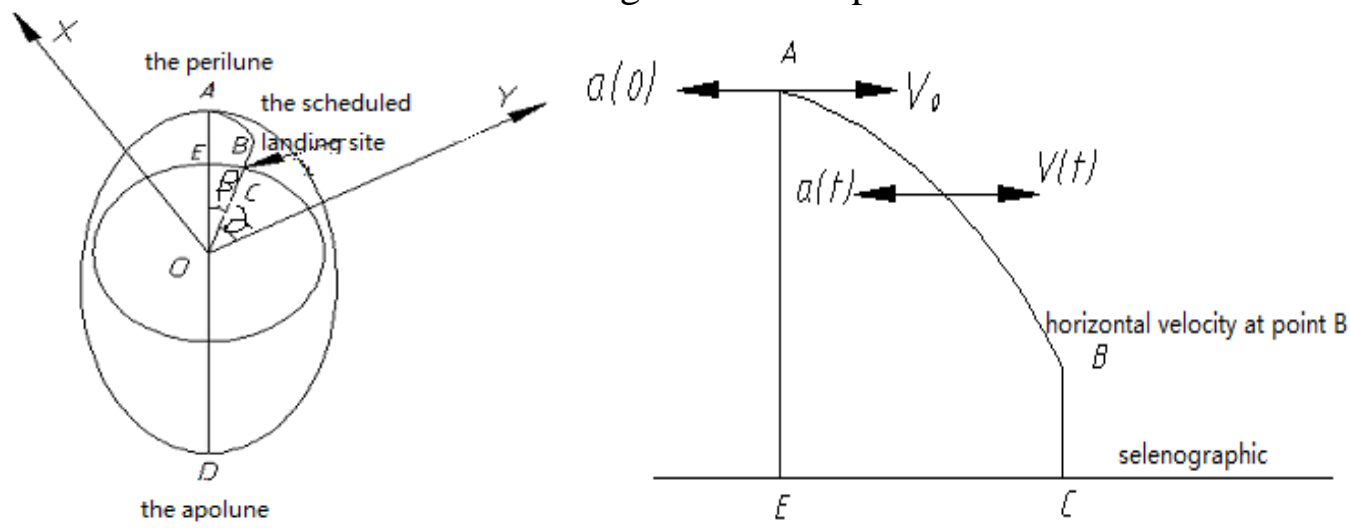

Fig. 1: Chang 'e 3 landing orbit plane Fig. 2: The second and third phase landing orbit plane According to the theorem of momentum we can get it as below:

$$
F_{t} \Delta t=m_{0} v_{0}, \quad F_{t}=v_{e} \dot{m}, \quad m(t)=m_{0}-\dot{m} t .
$$

And $\Delta t=750 \mathrm{~s}$ is the time difference from time $A$ to time $B$. The Newton's second law is

$$
F_{t}=m(t) \mathrm{a}(t) \text {. }
$$

so

$$
\mathrm{a}(t)=F_{t} / m(t), \quad v(t)=\int_{0}^{t} \mathrm{a}(t) d t, \quad s(t)=\int_{0}^{t} v(t) d t .
$$

From the above formulas we can get what as below:

$$
s(t)=\frac{v_{e} m_{0}}{\dot{m}}\left\{\left(m_{0}-m\right)\left[\ln \left(m_{0}-\dot{m} t\right)-1\right]-m_{0}\left(\ln m_{0}-1\right)\right\} .
$$

When $t=750 s$ (black 750 seconds), $s=809061 \mathrm{~m}$. That is to say $S$ is the approximate estimate length of $\operatorname{arc} B E$ in Fig. 1. We also can get that $\beta_{0}=s / R=0.466$, and when convert it to angle it equals to $\beta=26.7^{0}$, Then we can know the perilune's latitude is

$$
\alpha+\beta=70.82 N,
$$

So we can know the perilune location is $(19.51 W, 70.82 N)$ and the elevation is $15 \mathrm{~km}$. Because the perilune and the apolune is symmetrical about selenocenter, so the location of the apolune is $(160.49 E, 70.82 S)$ and the elevation is $100 \mathrm{~km}$. 


\section{The Chang 'e 3 optimal control strategy and its landing orbit}

Attitude control engine can realize adjustment of control automatically for all sorts of attitude through multiple pulse combination with numbers of engines which was installed around the Chang 'e 3 on the basis of a given thrust direction of the main reduction of engine.

Chang 'e 3 soft landing process has six stages[7], we can know the following control strategies through analysising the predetermined purpose and condition of various stages of the soft landing:

- Main reduction period (from $15000 m$ to $3000 m$ ): The speed direction of the Chang 'e 3 parallel to the moon surface on the horizontal direction when Chang 'e 3 out of the scheduled landing by assumption 2. The speed is very big and specific impulse of Chang 'e 3 is a constant value, the engine thrust is constant as long as the fuel consumption kilograms is constant per unit time, but it should content the limit of thrust parameters of Chang 'e 3.
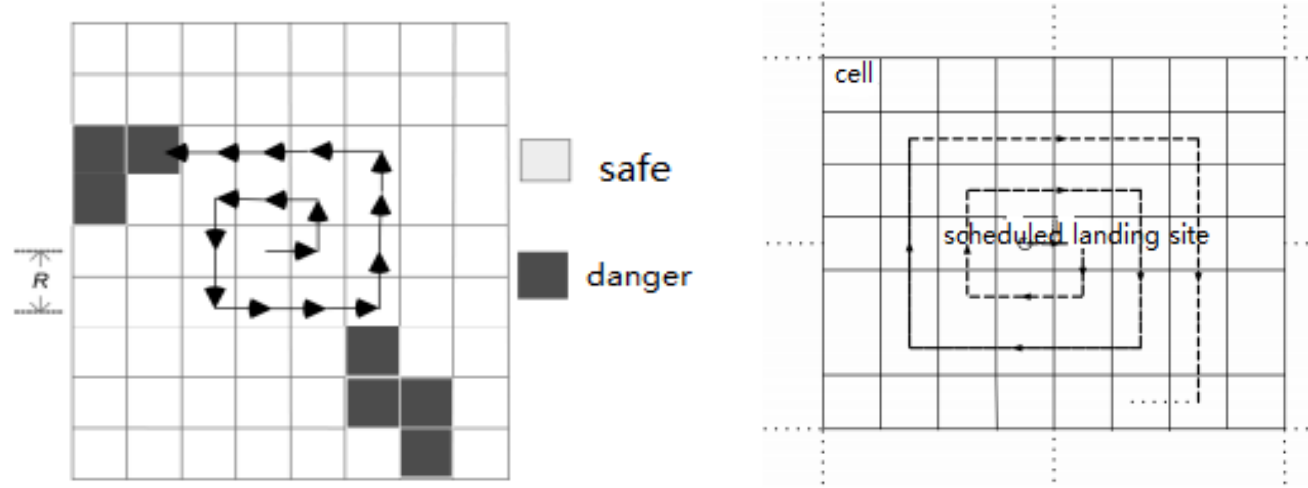

Fig. 3: Safe radius spiral search algorithm Fig. 4: The center spiral safe zone search algorithm

Fuel was consumed ( $\Delta m$ ) by the main thrust of engine reducer is equal if main force is constant in the same time. It can be proved by the following:

Further we can get it:

$$
\Delta m=\dot{m} \Delta t, \quad F_{t} \Delta t=m_{0} v_{0}, F_{t}=v_{\varepsilon} \dot{m},
$$

$$
\Delta m=\frac{m_{0} v_{0}}{v_{\varepsilon}}
$$

Due to the fuel consumption, the quality of Chang 'e 3 will constantly decrease and horizontal acceleration will gradually increase.

The engine in the sides can be closed in the vertical direction because there is no bigger gravity acceleration and no speed on the surface of the moon.

- Quick adjustment period (from $3000 \mathrm{~m}$ to $2400 \mathrm{~m}$ ): It makes main reduction thrust of engine vertical downward and the size is constant. Chang 'e 3 does decelerated motion with the increase of uniform acceleration only in the vertical direction and what the size of horizontal displacement is almost zero.

- Coarse obstacle avoidance section (from $2400 \mathrm{~m}$ to $100 \mathrm{~m}$ ): Keep the state of the third stage to make it hovering in the height of $100 \mathrm{~m}$. Selection method for design of obstacle recognition and safe landing area as follows:

1) Data processing. It mainly includes compensation for lander posture and translational velocity and turn slant distance information of each 'footprints' into vertical distance, then builds three-dimensional terrain elevation map on the basis of surveying coordinate systems;

2) Using least square method to fit the average slope in a certain unit area to complish slope construction;

3) Using the average slope to calculate average gradient and estimate barrier height of each cell;

4) Adopting the method of starting from the lander center clockwise spiral forward search (Fig. 3) until find the landing area which would accord with the requirement or is the optimal safe landing site and determine the safe landing points.

- Accurate obstacle avoidance section (from $100 \mathrm{~m}$ to $30 \mathrm{~m}$ ): The method to design accurate obstacle recognition and the method to select safe landing area is similar to design rough 
obstacle avoidance algorithm.

- Slow down period (from $30 \mathrm{~m}$ to $4 \mathrm{~m}$ ): Let the Chang 'e 3 do free fall first, then use the constant main thrust to eliminate the speed in the free fall.

- The period of free fall (from $4 m$ to lunar surface): Chang 'e 3 will do free fall before land on the moon surface.

Next, we will determine the landing trajectory of Chang 'e 3.

Assume $\beta(t)$ : the difference of latitude between Chang 'e 3 and the perilune in landing trajectory at moment $t$; $N(t)$ : latitude of Chang 'e 3 at moment $t$. From Fig. 4 we can know:

$$
\beta(t)=\frac{R s(t) \times 180}{\pi}, \quad N(t)=\alpha+\beta-\beta(t) .
$$

$N(t)$ reflects the latitude changes over time between Chang 'e 3 from the landing point in the perilune and reach above scheduled landing site. Because Chang 'e 3 runs on the longitude at scheduled landing place, so longitude $W$ is a constant and latitude of Chang 'e 3 can be determined by it's own ranging system at any time. The Chang 'e 3 orbit can be showed as horizontal displacement as follows since landing into orbit to reach the top of scheduled landing point by the formula (1):

$$
s(t)=\frac{v_{e} m_{0}}{\dot{m}}\left\{\left(m_{0}-m\right)\left[\ln \left(m_{0}-\dot{m} t\right)-1\right]-m_{0}\left(\ln m_{0}-1\right)\right\} .
$$

Whereby, longitude is

$W=$ constant (longitude of scheduled landing site) and latitude is

$$
N(t)=\alpha+\beta-\frac{R s(t) \times 180}{\pi}
$$

After the process, the horizontal displacement, longitude and latitude of reaching scheduled landing site won’t change any more.

\section{Summary}

The article aims at giving a control scheme of soft lunar landing and does research into estimation of orbit, the quality of burning up and the orbit control process of a soft landing on the basis of the characteristics of the different stages of a soft landing. It turned out operation process is clear and the data is reasonable on the basis of existing equipment works normally. Despite there are many idealize assumptions were proposed and exists numerical error in data processing of mathematical calculation process, but this algorithm is relatively simple and the actual process of a soft landing can be reflected objectively.

\section{References}

[1] M. Xu, J.F. Li, The optimal control of luner probe, Journal of Tsinghua University, 41 (2001) 87-89.

[2] B.L. Pieson, C.A. Kluever, Three-stage approach to optimal low-thrust earth-moon trajectories, Journal of Guidance, Control and Dynamics, 17 (1996) 1275-1282.

[3] W.L. Yang, The frozen orbits of the moon satellite, Journal of Astronautics, 29 (2008) 426-429.

[4] K.W. Tong, W. Liu, C.H. Gao, J.F. Wang, and D.J. Wang, Analysis of orbit perturbationsand frozen orbit for lunar satellite, Space craft engeering, 21 (2012) 1673-8748.

[5] Information on http://www.chinanews.com/mil/2013/12-12/5608941.shtml.

[6] Z.J. Chen, R.L. Zhang, P. Zhang, R. Zhou, The opportunities and challenges the spacecraft control is faced with, ACTA AUTOMATICA SINICA, 39 (2013) 703-710.

[7] Information on http://mil.news.sina.com.cn/2013-12-16/1027755135.html. 